\title{
Comparative effectiveness analysis of two regional analgesia techniques for the pain management of isolated multiple rib fractures
}

This article was published in the following Dove Press journal: Journal of Pain Research

\author{
Neal Lynch' \\ Kristin Salottolo ${ }^{1-4}$ \\ Krislyn Foster ${ }^{2}$ \\ Alessandro Orlando ${ }^{1-4}$ \\ Catherine Koola ${ }^{1-4}$ \\ Victor Portillo ${ }^{3}$ \\ Allen Tanner II \\ Charles W Mains ${ }^{4,5}$ \\ David Bar-Or ${ }^{1-4}$ \\ 'Trauma Services Department, Penrose- \\ St. Francis Health Services, Colorado \\ Springs, CO, USA; ${ }^{2}$ Swedish Medical \\ Center, Trauma Research Department, \\ Englewood, CO, USA; ${ }^{3}$ Medical City \\ Plano, Trauma Research Department, \\ Plano, TX, USA; ${ }^{4}$ St. Anthony Hospital, \\ Trauma Research Department, \\ Lakewood, CO, USA; ${ }^{5}$ Centura Health, \\ Trauma Services Department, \\ Englewood, CO, USA
}

Correspondence: David Bar-Or Swedish Medical Center, Trauma Research Department, 50I E Hampden Avenue, Room 4-454, Englewood, CO 80113 , USA

Tel +I 3037884089

Fax +I 3037884064

Email davidbme49@gmail.com
Background: Catheter-based regional analgesia has been proposed as an alternative to systemic analgesia for patients with multiple rib fractures (MRF). This study sought to compare the efficacy of regional techniques for decreasing pain and improving clinical outcomes.

Study design: This was a multi-institutional, retrospective cohort study of adult ( $\geq 18$ years) patients admitted to four nonacademic trauma centers over two years (from 07/1/2014 to 06/ 30/2016). Study inclusion was MRF ( $\geq 3$ fractures) with no other severe injuries. Two primary regional analgesia techniques were utilized and compared: continuous intercostal nerve blocks (CINB) and epidural (EPI) analgesia. The primary outcome, average pain scores on treatment, was examined using a repeated measures, linear regression mixed model. Secondary outcomes included hospital LOS, ICU LOS, ICU admission and hospital readmission, pulmonary complications, and incentive spirometry volumes during treatment, and were examined with univariate statistics.

Results: There were 339 patients with isolated MRF; 85 (25\%) required regional analgesia (CINB, $\mathrm{n}=41$; EPI, $\mathrm{n}=44$ ) and the remaining $75 \%$ received systemic analgesia only (IV, $\mathrm{n}=195 ; \mathrm{PO}, \mathrm{n}=59)$. There were demographic and clinical differences between regional analgesia and systemic analgesia groups; on the contrary, there were no demographic or clinical differences between the CINB and EPI groups. Adjusted pain scores were similar for the EPI and CINB groups ( 4.0 vs $4.4, p=0.49$ ). Secondary outcomes were worse in the EPI group compared to the CINB group: less improvement in incentive spirometry volume ( $p=0.004)$, longer ICU LOS $(p=0.03)$, longer hospital LOS $(p<0.001)$, and more ICU admission $(p<0.001)$.

Conclusion: In patients requiring regional analgesia, pain management was equivalent with CINB and EPI, but CINB was associated with significantly better clinical outcomes. CINB might offer an efficient alternative for pain control in patients with MRF.

Keywords: rib fractures, nerve block, epidural analgesia, trauma management

\section{Plain language summary}

Given the promising, yet limited, literature regarding the effectiveness of regional catheterbased analgesia, this study sought to compare the efficacy of regional analgesia techniques for decreasing pain and improving clinical outcomes. Two techniques were compared: continuous intercostal nerve blocks and epidural catheter analgesia. The results of this study demonstrated that patients with continuous intercostal nerve blocks reported similar self-reported pain scores compared to patients receiving epidural analgesia, but they had shorter hospital and ICU length of stay and better pulmonary function. Continuous 
intercostal nerve blocks offer a viable alternative for pain control in patients with multiple rib fractures, resulting in well controlled pain and favorable outcomes.

\section{Introduction}

Approximately $10 \%$ of the patients with blunt traumatic injury show evidence of rib fractures. ${ }^{1}$ In this population, about one-third of patients face significant complications as a result of pulmonary distress, with a mortality of $10 \%$ overall and $20 \%$ in the elderly. ${ }^{1-3}$ As the number of rib fractures increases, rates of mortality and morbidity exponentially increase. ${ }^{1}$

Adequate analgesia and pulmonary care are associated with enhanced functional capacity, improved respiratory mechanics, and the prevention of pulmonary complications. ${ }^{4}$

${ }^{6}$ Multimodal analgesia strategies are utilized for pain control, typically systemic pharmacologic agents including acetaminophen and non-steroidal anti-inflammatory drugs, with escalation to demand-only opioid analgesia.

Regional techniques have been shown to be associated with fewer side effects and greater efficacy when compared to systemic therapy, especially in patients with multiple rib fractures (MRF). ${ }^{7,8}$ The quality of evidence for other regional analgesia techniques is variable. The most frequently studied regional technique is epidural (EPI) catheter analgesia. Additional regional analgesia techniques that have shown promise include thoracic paravertebral blocks and continuous intercostal nerve blocks (CINB). ${ }^{9-12}$ The current joint practice management guideline for blunt thoracic trauma published by the Eastern Association for the Surgery of Trauma and Trauma Anesthesiology society found studies of poor quality and thus conditionally recommended epidural analgesia over nonregional modalities, with insufficient studies to make recommendations for CINB and other regional techniques. ${ }^{6}$

Given the promising, yet limited, literature regarding the effectiveness of regional catheter-based analgesia, we sought to compare the efficacy of regional analgesia techniques for decreasing pain and improving clinical outcomes.

\section{Methods}

\section{Aims and hypotheses}

The primary aim of the study was to examine the efficacy of regional analgesia techniques for decreasing pain in patients with MRF. The null hypothesis was that CINB analgesia would achieve similar analgesic effectiveness when compared EPI for acute pain management of patients with isolated MRF. The secondary aim was to compare regional analgesia techniques for improving clinical outcomes; the null hypothesis was that CINB and EPI analgesia techniques would report similar clinical outcomes.

\section{Setting, study design, and participants}

This multi-institutional, retrospective study was performed by the Injury Outcomes Network, a collaborative research network of six community-based, level I trauma centers. The study included adult $(\geq 18$ years) trauma patients admitted for MRF ( $\geq 3$ fractures) at four participating trauma centers, from $07 / 1 / 2015$ to $06 / 30 / 2016$ (hospitals A, B, C) and from 07/1/2014 to 06/30/2016 (hospital D).

Injury inclusion criteria were defined by ICD9 (807.09, 807.19) and ICD10 (S22.41-S22.43, S22.49) codes along with an abbreviated injury scale (AIS) score of 3 for the chest region. Patients were excluded if they had multitrauma, defined as an AIS score $\geq 3$ for body regions outside the chest. This study was approved by the institutional review boards at all participating centers and met the federal criteria for a waiver of informed consent.

\section{Treatment groups}

Patients were categorized into definitive treatment groups as follows:

- Regional analgesia: CINB and EPI analgesia

- Systemic: intravenous (IV) and oral (PO) pharmacologic agents.

In general, patients who were refractory to systemic analgesia were treated with regional analgesia (CINB or EPI) as a rescue technique. The agent, concentration, and flow rates for CINB and EPI were physician-dependent and varied by Richmond Agitation Sedation Scale scores and any previous experience with epidurals and nerve blocks, any allergies, and clinical presentation.

Patients receiving EPI were managed and overseen by anesthesia (anesthesiologist or CRNA) with procedural sedation and local anesthetic. EPI was generally administered with a locked electronic infusion device as a combination of bupivacaine $0.1 \%$ with $5 \mu \mathrm{g} / \mathrm{mL}$ of fentanyl and a rate of $4 \mathrm{~mL} / \mathrm{hr}$ as a continuous infusion through the catheter with portless tubing. Flow rates are titrated based on Richmond Agitation Sedation Scale score, systolic blood pressure, and respiratory rate. Coagulopathy is a patient exclusion for EPI. Moreover, patients requiring EPI have a pharmacologic thromboprophylaxis (PTP) hold for a safe window of usually $12 \mathrm{hrs}$ before the procedure, 
and then, PTP is provided as enoxaparin sodium $40 \mathrm{mg}$ once daily. Additional analgesia is provided for inadequate pain control or with IS volume $<10-15 \mathrm{cc} / \mathrm{kg}$. Incentive spirometry is encouraged 10 times every hour while awake. Patients should also have a 20-30 degree head of bed elevation.

The CINB consists of $5 \mathrm{~cm}$ and $7.5 \mathrm{~cm}$ multiple lumen catheters that are placed under local anesthetic and secured with tissue adhesive and sterile strips. Dressings are checked every $8 \mathrm{hrs}$ and are reinforced PRN. There are also routine checks for circulation, motion, and sensation. CINB medications are usually administered as bupivacaine ranging from $0.125 \%$ to $0.25 \%$ or ropivacaine ranging from $0.1 \%$ to $0.2 \%$. There are no patient exclusions for receiving CINB (including intubation and coagulopathy status). The upper range of ribs that can be treated is patient-specific including 3 or more ribs on a single side. If there are rib fractures bilaterally, then CINB can be done bilaterally.

\section{Study variables}

The following demographic, clinical, and outcome characteristics were obtained from the trauma registries: sex, age, cause of injury, ISS, comorbidities (yes/no), admission vital signs (systolic blood pressure, oxygen saturation, respiratory rate), ED disposition, hospital LOS, ICU LOS, ICU admission, readmission within 30 days, complications, and in-hospital mortality. Cause of injury was categorized as fall, vehicle-related, bicycle injury, or other. Complications were coded according to the National Trauma Data Bank data dictionary. ${ }^{13}$

The following clinical data were abstracted manually from patient health records: number of fractures (dichotomized as $3-5$ and $\geq 6$ fractures), type of fracture (unilateral vs bilateral), definitive analgesic treatment start time, and definitive analgesic treatment stop time. Patientreported pain scores, ranging from 0 to 10 on the numeric rating scale (lower scores indicate lower pain), and incentive spirometry volumes were also manually abstracted from patient health records at two time points: pretreatment (last recorded volume before definitive treatment was initiated) and posttreatment (last recorded volume before discharge). Incentive spirometry volumes were further categorized as low capacity $<1$ L vs normal capacity $\geq 1$ L. The fraction of inspired oxygen (FiO2) was collected longitudinally for ventilated patients. We also abstracted arterial blood gases, but they were only recorded in $8 \%$ and were not examined.

\section{Outcome measures}

The primary outcome was average pain during analgesic treatment. Secondary outcomes included average change in pain scores on treatment minus pretreatment (mean scores recorded $24 \mathrm{hrs}$ before treatment), hospital LOS, ICU LOS, and mean change in incentive spirometry volume from pretreatment to hospital discharge, ICU admission, hospital readmission within 30 days, in-hospital mortality, and complications: a) all complications; b) pulmonary complications (pneumonia, acute respiratory distress syndrome, respiratory failure); c) unintended intubation; d) procedural complications.

\section{Statistical analyses}

Differences in demographic and clinical characteristics across treatment groups (CINB, EPI, IV, and PO analgesia) were evaluated with Fisher's exact tests or generalized linear models.

Study outcomes were analyzed in the subset of patients requiring regional analgesia (CINB vs EPI), as these patients were not adequately managed with systemic analgesia and required a rescue technique. Pain was examined as the average pain score for each $24 \mathrm{hr}$ period and evaluated by treatment group (CINB vs EPI) during the first $96 \mathrm{hrs}$ on treatment using a repeated measures, linear regression mixed model with a compound symmetry covariance structure. Only the first four days of pain scores during treatment were examined because, after day four, there was a sharp decrease in the number of patients who remained on treatment $(<50 \%)$. Secondarily, pain was examined as the change in average pain score on treatment minus the average pretreatment (systemic analgesia only) pain score and was evaluated by treatment group (CINB vs EPI) using an ANCOVA model. Results for the primary outcome are reported before adjustment and after adjustment for variables that met a $p$-value $<0.20$ in univariate analysis (ISS, high rib fracture count, presence of a comorbidity).

Secondary outcomes were evaluated with Fisher's exact tests (dichotomous end points), Student's $t$-tests (continuous endpoints), and Wilcoxon rank sum tests (ordinal end points).

\section{Results}

\section{Patient characteristics}

There were 339 patients with isolated MRF; 85 patients required regional analgesia $(C I N B, n=41 ; E P I, n=44)$. The remaining $75 \%$ of the patients were successfully treated with 
systemic pharmacologic agents (IV, n=195; PO, $n=59$ ). Differences in patients requiring regional vs systemic analgesia can be found in Table 1. Patients requiring regional analgesia were older, had more rib fractures, were more likely to have bilateral fractures, and present with an abnormal respiratory rate. As anticipated, the time to initiate definitive analgesia was significantly longer in those who required regional analgesia as a rescue technique compared to patients who were successfully treated with systemic pharmacologic agents.

Table 1 also compares demographics and clinical characteristics for CINB vs EPI; there were no demographic or clinical differences observed. High rib fracture count, ISS, and the presence of a comorbidity met $p<0.20$ and were adjusted for in the final model. Low baseline oxygen saturation also met the $p<0.20$ entry criteria but was not adjusted for as there were more than $20 \%$ missing data in the EPI group. Three (7\%) patients with CINB were intubated, all before CINB. Six (14\%) patients receiving EPI were intubated, 3 before and 3 after EPI.

There were no differences in the number of hours to definitive treatment for CINB vs EPI $(p=0.09)$, Table 1. Patients receiving EPI were on treatment for significantly longer than patients receiving CINB (121 h vs $63 \mathrm{~h}$, $p=0.005)$. However, $27(66 \%)$ patients were discharged with the CINB in place.

\section{Pain}

There was an average of 15.6 pain scores per patient on CINB and an average of 29.8 pain scores per patient on EPI, resulting in a similar pain measurement frequency of q4 hrs for both treatments. In the $24 \mathrm{hr}$ period before definitive treatment, there was an average number of 5.3 pain scores for EPI patients and 5.0 pain scores for CINB patients.

Pain scores decreased with regional analgesia during the first four days on treatment (Figure 1). Before

Table I Differences in demographics and clinical characteristics

\begin{tabular}{|c|c|c|c|c|c|c|}
\hline Variable & $\begin{array}{l}\text { CINB } \\
(n=4 I)\end{array}$ & $\begin{array}{l}\text { EPI } \\
(n=44)\end{array}$ & $\begin{array}{l}\text { IV } \\
(n=195)\end{array}$ & $\begin{array}{l}\text { PO } \\
(n=59)\end{array}$ & $\begin{array}{l}\text { p-Value } \\
\text { (across }^{\text {treatments) }}\end{array}$ & $\begin{array}{l}p-\text { Value } \\
\text { (CINB vs } \\
\text { EPI)c }\end{array}$ \\
\hline Male gender, n (\%) & $27(65.8)$ & $27(61.4)$ & I 38 (70.8) & $38(64.4)$ & 0.57 & 0.67 \\
\hline Age $\geq 65, n(\%)$ & $28(68.3)$ & $31(70.5)$ & $85(43.6)$ & $32(54.2)$ & 0.001 & 0.83 \\
\hline$\geq 6$ rib fractures, $n(\%)$ & $30(73.2)$ & $25(56.8)$ & $54(27.7)$ & $9(24.3)^{b}$ & $<0.001$ & 0.11 \\
\hline Number of fractures, mean (SD) & $6.4(1.8)$ & $6.2(2.5)$ & $5.0(2.4)$ & $4.7(1.3)^{b}$ & $<0.001$ & 0.62 \\
\hline Unilateral fractures, n (\%) & $32(78.1)$ & $33(75.0)$ & $172(88.2)$ & $38(100)^{b}$ & 0.003 & 0.74 \\
\hline ISS, mean (SD) & II.I (3.0) & $11.8(2.5)$ & $11.7(3.0)$ & $12.0(4.5)$ & 0.55 & 0.20 \\
\hline Cause of injury, $n$ (\%) & & & & & 0.46 & 0.76 \\
\hline Fall & $19(46.3)$ & $20(45.5)$ & 91 (46.7) & $29(49.2)$ & & \\
\hline Vehicular & $18(43.9)$ & $20(45.5)$ & 66 (33.9) & $23(39.9)$ & & \\
\hline Bike & I (2.4) & $0(0)$ & $17(8.7)$ & $3(5.1)$ & & \\
\hline Other & $3(7.2)$ & $4(9.1)$ & $21(10.8)$ & $4(6.8)$ & & \\
\hline Any comorbidity, n (\%) & $32(78.1)$ & $40(90.9)$ & $167(85.6)$ & $54(91.5)$ & 0.20 & 0.10 \\
\hline Baseline IS $<I \mathrm{~L}$ & $94.9(37)$ & $36.7(11)$ & $21.3(17)$ & $18.2(2)$ & $<0.001$ & $<0.001$ \\
\hline Baseline oxygen sat $<92 \%$ & $8(20.5)$ & $2(7.4)^{\mathrm{b}}$ & $13(10.0)^{\mathrm{b}}$ & $5(I I . I)^{b}$ & 0.28 & 0.18 \\
\hline Mechanically ventilated & $3(7.3)$ & $5(11.4)$ & $7(3.6)$ & $6(10.2)$ & 0.12 & 0.52 \\
\hline $\mathrm{FiO} 2$, mean $(\mathrm{SD})$ & $50.7(19)^{\mathrm{b}}$ & $60.0(3 \mathrm{I})^{\mathrm{b}}$ & $47.8(16)$ & $59(15)^{b}$ & 0.19 & 0.55 \\
\hline Baseline SBP $<90 \mathrm{~mm} \mathrm{Hg}$ & $0(0)^{b}$ & $2(4.8)$ & $4(2.1)$ & $3(5.1)$ & 0.46 & 1.00 \\
\hline Baseline heart rate $>120$ beats $/ \mathrm{min}$ & I (2.6) & $2(4.6)$ & $5(2.8)$ & $0(0)$ & 0.70 & 1.00 \\
\hline Baseline $R R<12$ or $>20$ & $27(65.9)$ & $26(59.1)$ & $93(47.7)$ & $21(35.6)$ & 0.01 & 0.52 \\
\hline Hours (IQR) to definitive analgesia ${ }^{a}$ & $41.3(36.7)$ & $30.1(38.7)$ & $0.5(12.6)$ & $2.0(12.3)$ & $<0.001$ & 0.09 \\
\hline Hours (IQR) on definitive analgesia ${ }^{a}$ & $63.1(82.3)$ & $121.2(62.2)$ & $48.7(66.7)$ & NR & $<0.001$ & 0.005 \\
\hline Mean (SD) pain before definitive analgesia & $5.2(2.0)$ & $5.0(1.8)$ & $5.3(2.6)$ & $4.2(1.7)$ & 0.06 & 0.58 \\
\hline Mean (SD) pain with definitive analgesia & $3.8(2.1)$ & $3.8(1.5)$ & 4.9 (1.9) & NR & $<0.001$ & 0.96 \\
\hline
\end{tabular}

Notes: ${ }^{a}$ median, interquartile range. ${ }^{b}$ Greater than $20 \%$ missing data; ${ }^{c} p$-Values were calculated with chi-square test for categorical variables, generalized linear model for means, Wilcoxon rank sum tests for medians. Bolding denotes statistical significance.

Abbreviations: CINB, continuous intercostal nerve block; EPI, epidural analgesia; IS, incentive spirometry; ISS, injury severity scale; IV, intravenous pharmacologic agents; $\mathrm{PO}$, per os (oral pharmacologic agents); SBP, systolic blood pressure; RR, respiratory rate; NR, not reported; FiO2, fraction of inspired oxygen. 


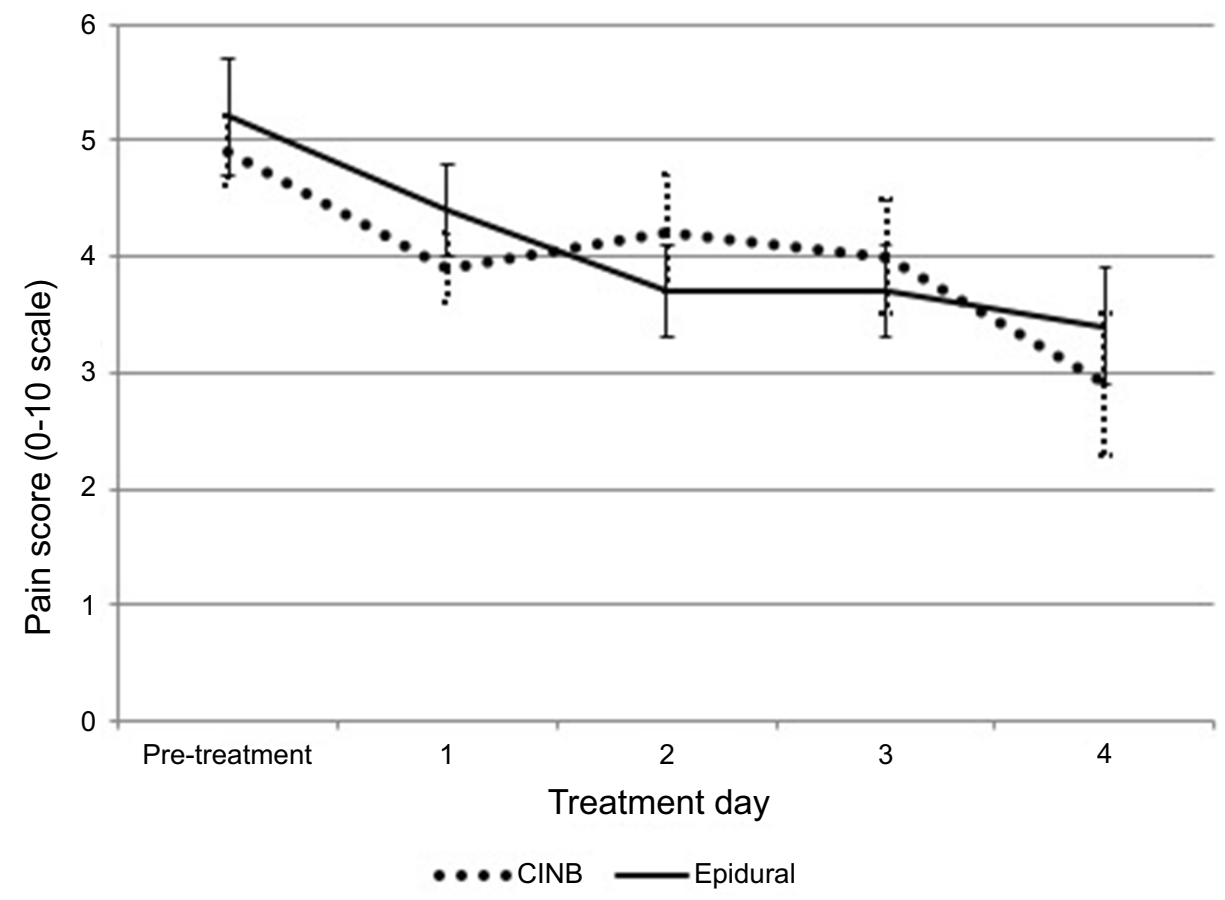

Figure I Average self-reported pain on the 0-10 numeric rating scale during treatment days I through 4, by analgesic treatment modality. Error bars represent standard error. Abbreviation: CINB, continuous intercostal nerve block.

adjustment, the EPI and CINB groups reported identical mean pain scores ( $p=0.94$, Table 2$)$. After adjustment for the number of rib fractures, ISS, and comorbidity, there were no differences in pain scores between the EPI and CINB groups (4.0 vs $4.4, p=0.49$ ). High rib fracture count was the only covariate that was significantly associated with pain scores in the adjusted model $(p=0.04)$.

The change in pain score with regional analgesia was similar for EPI and CINB groups, both before $(p=0.55)$ and after adjustment $(p=0.90)$, Table 2 and Figure 2 .

\section{Secondary outcomes}

Analysis of secondary outcomes revealed a significant difference between CINB and EPI groups for hospital LOS, ICU admission, and incentive spirometry change (Table 3). EPI patients had significantly longer hospital LOS, ICU LOS, and greater rate of ICU admission compared to CINB. Pulmonary function as measured by incentive spirometry volumes improved more with CINB $(p=0.004)$, and the CINB group reported a smaller percentage of patients with low incentive spirometry volume $<1 \mathrm{~L}$ during treatment, despite having a significantly higher proportion with incentive spirometry volumes $<1 \mathrm{~L}$ pretreatment. There were no differences between groups in the development of any complication $(p=0.17)$ or specific complications. There were no procedural or device-related complications reported for either group.

Additionally, we also compared the hospital LOS for CINB patients who had the catheter in place at the time of discharge versus patients who had the catheter removed. As expected, LOS was shorter when CINB was in place ( 5.6 days vs 8.5 days, $p=0.04$ ), and there was no difference in pain scores on treatment between groups $(p=0.22)$.

\section{Discussion}

The primary objective of this study was to compare the effectiveness of two regional analgesia techniques for patients with isolated MRF. Our study demonstrated that self-reported pain was similar to EPI compared to CINB, both before and after adjustment. Pain scores are considered controlled with acute pain management for scores $<4$ on a $0-10$ scale. ${ }^{14}$ Treatment with EPI and CINB resulted in well-controlled pain, with an average pain score of approximately 3.8 in both groups, down from the pretreatment pain scores of 5 or greater. More importantly, the secondary outcomes of this study demonstrated that the EPI group had worse clinical outcomes, including more ICU admission, a two-fold increase in ICU LOS and hospital LOS, and less improvement in incentive spirometry than patients treated with CINB. A primary outcome of pain may not be the 
Table 2 Primary outcome: pain scores during treatment

\begin{tabular}{|l|l|l|l|}
\hline Model & CINB (n=4I) & EPI (n=44) & p-Value \\
\hline \multicolumn{2}{|l|}{ Average pain score on treatment (SE) } & $3.83(0.25)$ & 0.94 \\
\hline Unadjusted & $3.85(0.2 \mathrm{I})$ & $4.02(0.42)$ & 0.49 \\
Adjusted $^{\mathrm{a}}$ & $4.37(0.38)$ & $-1.15(-1.83,-0.47)$ & 0.55 \\
\hline Mean change in pain from pretreatment (95\% Cl) & $-0.95(-1.80,-0.10)$ & 0.90 \\
\hline Unadjusted $_{\text {Adjusted }^{\mathrm{a}}}$ & $-1.43(-2.09,-0.78)$ & $-1.01(-1.81,0.22)$ & \\
\hline
\end{tabular}

Notes: ${ }^{2}$ Adjusted for comorbidity (yes/no), injury severity score, and high rib fracture count. Pain score on treatment examined with a repeated measures mixed model for average scores on treatment days $\mathrm{I}-4$, with a compound symmetry covariance matrix. Change in pain scores examined with a general linear model for average score on treatment-average score pretreatment.

Abbreviations: CINB, continuous intercostal nerve block; EPI, epidural analgesia; SE, standard error.

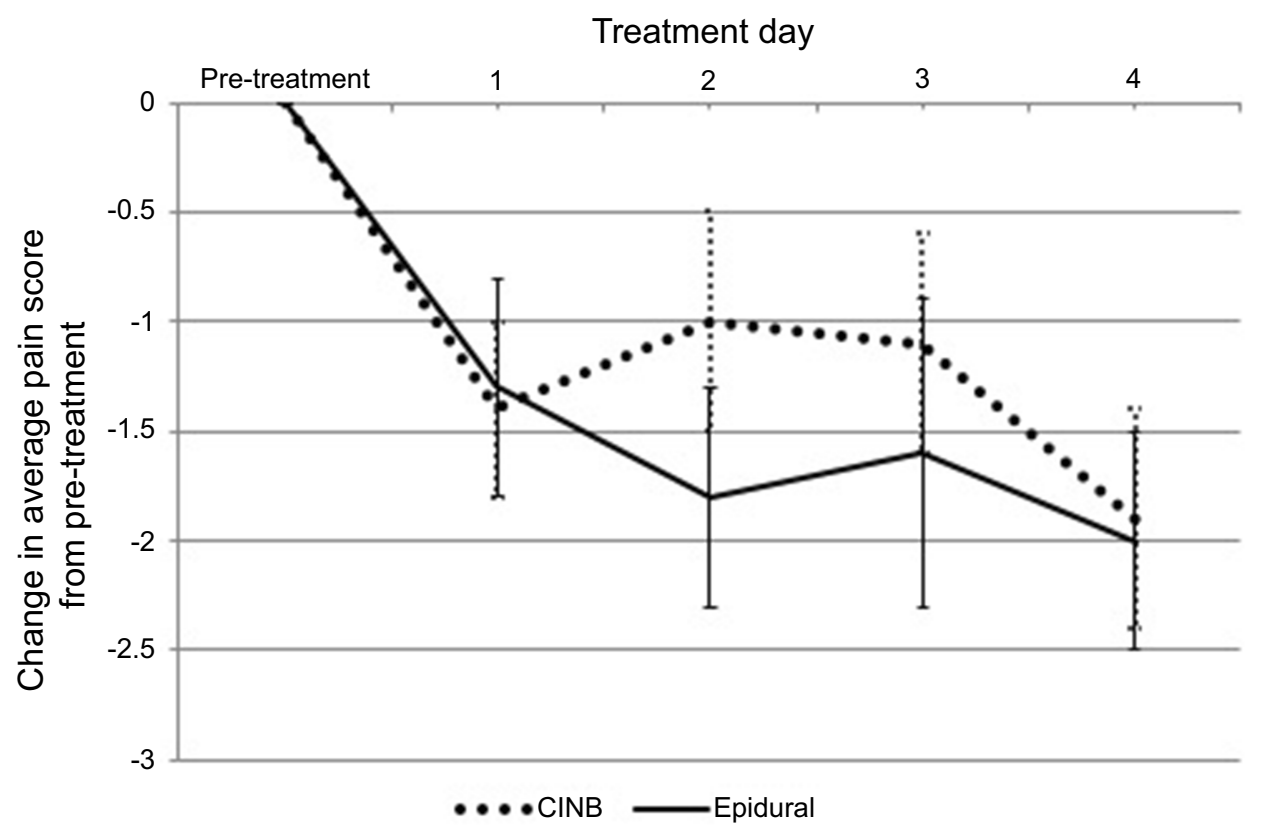

Figure 2 Change in average self-reported pain (on the 0-10 numeric rating scale) during regional analgesia, from pre-regional analgesia. Error bars represent standard error. Abbreviation: CINB, continuous intercostal nerve block.

Table 3 Secondary outcomes

\begin{tabular}{|c|c|c|c|}
\hline Variable & CINB (n=4I) & EPI $(n=44)$ & $p$-Value \\
\hline Any complication, n (\%) & $9.8(4)$ & $20.5(9)$ & 0.17 \\
\hline Pulmonary complication, n (\%) & $4.9(2)$ & II.4 (5) & 0.44 \\
\hline Procedural complication, n (\%) & $0(0)$ & $0(0)$ & - \\
\hline Unplanned ICU admission, n (\%) & $0(0)$ & $2.3(1)$ & 1.0 \\
\hline Unintended intubation, $\mathrm{n}(\%)$ & $4.9(2)$ & $2.3(1)$ & 0.61 \\
\hline Median change in IS, L (IQR) & $0.70(0.25-1.20)$ & $0.08(0-0.50)$ & 0.004 \\
\hline Readmission, n (\%) & $2.4(I)$ & $7.0(3)$ & 0.62 \\
\hline ICU admission, n (\%) & $46.3(19)$ & $86.4(38)$ & $<0.001$ \\
\hline ICU LOS, mean (SD) & $4.0(4.3)$ & $6.5(3.5)$ & 0.03 \\
\hline Hospital LOS, mean (SD) & $6.6(3.8)$ & $10.1(4.3)$ & $<0.001$ \\
\hline Mortality, n (\%) & $0(0)$ & $0(0)$ & 1.00 \\
\hline
\end{tabular}

Notes: ${ }^{2}$-values were calculated with Fisher's exact test for categorical variables, Student's $t$-tests for continuous variables, and Wilcoxon rank sum tests for medians. Bolding denotes statistical significance.

Abbreviations: CINB, continuous intercostal nerve block; EPI, epidural analgesia; IQR, interquartile range; IS, incentive spirometry. 
most clinically relevant if decreased pain does not result in improved pulmonary function and shorter time in hospital.

Despite its retrospective design, the current study adds necessary information to the limited literature examining regional analgesia techniques for MRF. Three studies comprise the limited literature comparing CINB and EPI techniques. In 2011, Truitt and colleagues conducted a prospective study demonstrating significant improvements in pulmonary function and pain control with CINB, with a shorter hospital stay when compared to a historical sample of patients receiving EPI. ${ }^{12}$ Also in 2011, Hashezmedeh et al compared EPI vs CINB among 60 patients with MRF. EPA was superior to CINB for pain (days 1 through 3), and ICU and hospital LOS were both shorter with EPI treatment. ${ }^{10}$ Most recently in 2015, Britt et al conducted a retrospective comparison of rib fracture patients receiving CINB versus EPI and demonstrated significantly reduced hospital LOS for the CINB group but no difference in respiratory complications, ventilator days, or ICU LOS. ${ }^{9}$ As such, one catheter-based method is not consistently more effective than the other.

Our study supports the findings by Truitt $^{12}$ and Britt, ${ }^{9}$ demonstrating a shorter hospital LOS for CINB over EPI. Our study methodology can be considered superior to that by Truitt et al because the authors compared CINB to a historical EPI control group admitted two years prior. In the prospective CINB sample, hospital LOS was only 2.9 days and $80 \%$ were discharged home with catheters in place, which was a shorter LOS and a higher proportion discharged with CINB than our study reports. The LOS presented by Britt et al were almost identical to this study: CINB (6.9 vs 6.6 days, respectively) and EPI (9.7 vs 10.1 days, respectively). A shorter hospital LOS may be the result of patients being able to be discharged with the CINB still in place, providing no break in pain management. CINB also has the advantage of being easily placed at the beside by advanced practice personnel under local anesthesia with minimal complications, thus not requiring OR time or anesthesia involvement.

We identified two randomized studies that compared CINB with EPI following thoracotomy; in this condition, there was no consensus on optimal management. ${ }^{15,16}$ Luketich and colleagues designed a trial to evaluate postoperative pain for 124 patients randomized to EPI and CINB and found no difference between groups for pain or pulmonary function, hospital LOS, and complications. ${ }^{16}$ In the study by Debreceni et al, 50 patients were randomized to EPI or CINB. Respiratory function and pain were evaluated postoperatively. Pain in the early postoperative period was better controlled with EPI than CINB, with no significant difference in respiratory function between groups. ${ }^{15}$ It is difficult to extend the results of these two conflicting regional analgesia trials in the setting of thoracotomy to the setting of MRF.

Study limitations are as follows: primarily, this was an observational retrospective cohort study. A prospective, appropriately powered study is required to determine the optimal management of acute pain following MRF, with an appropriate study end point. Second, we excluded patients with significant extrathoracic injuries, reducing the generalizability of our findings. EPA is more likely to be contraindicated in patients with competing injuries. Third, we did not collect total opioid consumption, which is an important outcome since the escalating opioid crisis has led to calls to action to reduce opioid consumption. ${ }^{17}$ While not studied here, we believe this technique has the ability to decrease opioid use and be a key component in the battle of the opioid crisis with these select but significant patients. Finally, our study did not examine other regional and local modes of analgesia such as thoracic paravertebral blocks or non-CINB. These modalities still need to be demonstrated in sufficient quality and quantity before they can be recommended as more effective than systemic analgesia.

\section{Conclusion}

This study demonstrated that regional analgesia techniques of CINB and EPI are associated with similar pain control following MRF, but that CINB is superior to EPI in providing favorable clinical and functional outcomes. CINB appears to be an alternative to EPA resulting in wellcontrolled pain, shorter hospital LOS, and improved pulmonary function. Given the promising nature of CINB and the rare reported incidence of complications in the literature, a randomized controlled trial could provide the thorough evaluation and evidence needed to elevate this novel treatment to a standard of care for managing pain in the setting of MRF.

\section{Abbreviation list}

AIS, abbreviated injury scale; CINB, continuous intercostal nerve blocks; ED, emergency department; EPI, Epidural catheters; HLOS, hospital length of stay; ICULOS, Intensive care unit length of stay; ISS, injury severity score; IV, intravenous; MRF, multiple rib fractures; PO, per os (oral). 


\section{Ethics approval and consent}

The study was approved from the Institutional Review Boards at each respective facility; informed consent was waived. Reference numbers: Swedish Medical Center: 1046117; St. Anthony Hospital: 1046983; Penrose Hospital: 1046983 (same IRB as St.Anthony); Medical City Plano: 1058377.

\section{Acknowledgments}

We would like to acknowledge the study coordinators at each of the participating trauma centers for assistance with data abstraction: Diane Redmond (Penrose-St. Francis); Jennifer Pekarek (St. Anthony Hospital); Carolyn Blue (Swedish Medical Center); and Kathy Rodkey (Medical City Plano). The study was investigator initiated. Internal funding was provided by Swedish Medical Center, St. Anthony Hospital, Penrose Hospital, Medical City Plano, Research Medical Center (Kansas City, MO), and Wesley Medical Center (Wichita, KS).

\section{Author contributions}

All authors contributed to data analysis, drafting and revising the article, gave final approval of the version to be published, and agree to be accountable for all aspects of the work.

\section{Disclosure}

The authors report no conflicts of interest in this work.

\section{References}

1. Flagel BT, Luchette FA, Reed RL, et al. Half-a-dozen ribs: the breakpoint for mortality. Surgery. 2005;138(4):717-723; discussion 723-715. doi:10.1016/j.surg.2005.07.022

2. Bulger EM, Arneson MA, Mock CN, Jurkovich GJ. Rib fractures in the elderly. J Trauma. 2000;48(6):1040-1046; discussion 1046-1047.

3. Bergeron E, Lavoie A, Clas D, et al. Elderly trauma patients with rib fractures are at greater risk of death and pneumonia. J Trauma. 2003;54(3):478-485. doi:10.1097/01.TA.0000037095.83469.4C

4. Brasel KJ, Moore EE, Albrecht RA, et al. Western trauma association critical decisions in trauma: management of rib fractures. J Trauma Acute Care Surg. 2017;82(1):200-203. doi:10.1097/ TA.0000000000001301

Journal of Pain Research

\section{Publish your work in this journal}

The Journal of Pain Research is an international, peer reviewed, open access, online journal that welcomes laboratory and clinical findings in the fields of pain research and the prevention and management of pain. Original research, reviews, symposium reports, hypothesis formation and commentaries are all considered for publication. The manuscript
5. Brown SD, Walters MR. Patients with rib fractures: use of incentive spirometry volumes to guide care. J Trauma Nurs. 2012;19(2):89-91; quiz 92-83. doi:10.1097/JTN.0b013e31825629ee

6. Galvagno SM Jr., Smith CE, Varon AJ, et al. Pain management for blunt thoracic trauma: a joint practice management guideline from the Eastern Association for the Surgery of Trauma and Trauma Anesthesiology Society. J Trauma Acute Care Surg. 2016;81 (5):936-951. doi:10.1097/TA.0000000000001209

7. Karmakar MK, Ho AM. Acute pain management of patients with multiple fractured ribs. $J$ Trauma. 2003;54(3):615-625. doi:10.1097/ 01.TA.0000053197.40145.62

8. Ho AM, Karmakar MK, Critchley LA. Acute pain management of patients with multiple fractured ribs: a focus on regional techniques. Curr Opin Crit Care. 2011;17(4):323-327. doi:10.1097/MCC. 0b013e328348bf6f

9. Britt T, Sturm R, Ricardi R, Labond V. Comparative evaluation of continuous intercostal nerve block or epidural analgesia on the rate of respiratory complications, intensive care unit, and hospital stay following traumatic rib fractures: a retrospective review. Local Reg Anesth. 2015;8:79-84. doi:10.2147/LRA.S80498

10. Hashemzadeh S, Hashemzadeh K, Hosseinzadeh H, Aligholipour Maleki R, Golzari SE. Comparison thoracic epidural and intercostal block to improve ventilation parameters and reduce pain in patients with multiple rib fractures. J Cardiovasc Thorac Res. 2011;3 (3):87-91. doi:10.5681/jevtr.2011.019

11. Truitt MS, Mooty RC, Amos J, Lorenzo M, Mangram A, Dunn E. Out with the old, in with the new: a novel approach to treating pain associated with rib fractures. World J Surg. 2010;34(10):2359-2362. doi:10.1007/s00268-010-0651-9

12. Truitt MS, Murry J, Amos J, et al. Continuous intercostal nerve blockade for rib fractures: ready for primetime? J Trauma. 2011;71 (6):1548-1552; discussion 1552. doi:10.1097/TA.0b013e31823c96e0

13. The Committee on Trauma. National Trauma Data Standard Data Dictionary. 2019 Admissions. 2018 ed.; American College of Surgeons. Available from: https://www.facs.org/ /media/files/quality $\% 20 \mathrm{programs} / \mathrm{trauma} / \mathrm{ntdb} / \mathrm{ntds} /$ data\%20dictionaries/ntdb_data_dic tionary_2019_revision.ashx. Accessed March 12, 2019.

14. Witt CE, Bulger EM. Comprehensive approach to the management of the patient with multiple rib fractures: a review and introduction of a bundled rib fracture management protocol. Trauma Surg Acute Care Open. 2017;2(1):e000064. doi:10.1 136/tsaco-2016-000064

15. Debreceni G, Molnar Z, Szelig L, Molnar TF. Continuous epidural or intercostal analgesia following thoracotomy: a prospective randomized double-blind clinical trial. Acta Anaesthesiol Scand. 2003;47 (9):1091-1095.

16. Luketich JD, Land SR, Sullivan EA, et al. Thoracic epidural versus intercostal nerve catheter plus patient-controlled analg esia: a randomized study. Ann Thorac Surg. 2005;79 (6):1845-1849; discussion 1849-1850. doi:10.1016/j.athor acsur.2004.10.055

17. The Opioid Crisis. Available from: https://www.whitehouse.gov/ opioids/. Accessed May 23, 2018. management system is completely online and includes a very quick and fair peer-review system, which is all easy to use. Visit http:// www.dovepress.com/testimonials.php to read real quotes from published authors. 